\title{
DETECTION OF SUPERHUMPS DURING THE OUTBURST OF BC UMA
}

\author{
CHATIEF KUNJAYA \\ Bosscha Observatory, Indonesia \\ AND \\ TAICHI KATO AND RYUKO HIRATA \\ Kyoto University, Japan
}

BC UMa was observed during its rare outburst in 1994. The superhumps with period 0.0619 day are clearly apparent, accompanied by secondary superhumps. This confirms the classification of BC UMa as an SU UMa type dwarf nova. The maximum brightness is $12.5 \mathrm{mag}$, which is about six magnitude brighter than quiescence phase, and it makes $\mathrm{BC}$ UMa be classified as a TOAD (Tremendous Outburst Amplitude Dwarf-nova). The superoutburst's magnitude was fainter than the preceeding normal outburst in September 1990. This faint superoutburst is uncommon among SU UMa stars and also among TOADs. The period analysis of the lightcurve taken after the end of the outburst yields the period of 0.0618 day which is shows that late superhumps is in operation. 